\title{
Continuous optimisation techniques for optimal aiming strategies in solar power tower plants
}

\author{
Thomas Ashley ${ }^{\mathrm{a}, *}$, Emilio Carrizosa ${ }^{\mathrm{a}}$, Enrique Fernández-Cara ${ }^{\mathrm{b}}$ \\ ${ }^{a}$ IMUS - Instituto de Matemáticas de la Universidad de Sevilla, Spain \\ ${ }^{\mathrm{b}}$ Dep. EDAN and IMUS, Universidad de Sevilla, Spain
}

\section{A R T I C L E I N F O}

\section{Keywords:}

Solar energy

Bi-objective continuous optimization

Aiming strategy

Operations research

\begin{abstract}
A B S T R A C T
Optimising the aiming strategy is crucial for Solar Power Tower plants, in order to maximise the energy generated, whilst also preventing catastrophic damage to receiver components. In this work, a bi-objective optimisation model is developed to find optimal aiming strategies for a Solar Power Tower plant. The primary objective to maximise the radiation captured by the receiver is offset by a secondary objective to minimise the deviation from a desired target distribution, which is designed by solar plant operators to improve plant efficiency. A numerical method is proposed to solve the optimisation problem, and an illustrative example is presented to show functionality of the model and the numerical method. Conclusions are drawn on the model presented, extensions are considered and current work is discussed.
\end{abstract}

\section{Introduction}

The optimisation of Solar Power Tower (SPT) plant operations and subsystems can lead to improved efficiency and successful implementation of the technology, and is a key factor in future development. In a SPT plant, a type of Concentrating Solar Power (CSP) technology, the incident solar radiation is focused onto a receiver mounted atop a tower and the resultant heat used to drive a conventional generator. Efficiency in a SPT plant is highly dependent on construction and the effective management of various subsystems.

Indeed, CSP plants include subsystems that are open to optimisation in both construction and operation, and have featured prominently in recent literature, for example Abu-hamdeh and Alnefaie (2016), Baños et al. (2011), Carrizosa et al. (2015a), Carrizosa et al. (2015b), Carrizosa et al. (2017), and Morais et al. (2010). A SPT plant utilises a field of heliostats to concentrate the incident radiation onto the receiver surface, where the focus point of each heliostat is independent from the others. The position of focal points for all heliostats in the field, known as the aiming strategy, has been shown in previous research to be an important factor in plant operation, see Astolfi et al. (2016).

Such research has involved the use of various methods for the optimisation of aiming strategies, namely Linear Programming (Ashley et al., 2017), Parallel Teaching-Learning Algorithms (Cruz et al., 2016) and an Ant Colony optimisation (Belhomme et al., 2014).
However, maximising only radiation captured is not an adequate criterion, since steep thermal gradients or focused spikes are capable of causing permanent damage to receiver components or lowering efficiency of thermal transfer (Relloso and García, 2015; Yu et al., 2014). The optimisation problem can therefore consider penalty terms or secondary objectives to minimise such phenomena. In other words, the problem under consideration is a multi-objective optimisation problem, see Przybylski and Gandibleux (2017), and Tawhid and Savsani (2018).

Binary integer programming was applied in Ashley et al. (2017) to optimise the aiming strategy for a SPT plant at a fixed point in time, by restricting the aiming points to a predefined set of possible options. If the number of heliostats or aim points is high, the problem dimensionality may become too large, therefore the restriction of aiming point location to a limited predefined set reduced the problem dimensionality to manageable levels. Whilst this method allowed rapid analysis of optimal aiming strategies, it also limited the possible solution space to the selected grid of aiming points.

A more accurate approach is to allow the aiming point variables to take any value and be continuously differentiable across the receiver surface. This is equivalent to defining a separate aiming point for each heliostat in the field, within the set created by the boundaries of the receiver dimensions. This allows the simulation of SPT plant operations to better reflect real-life conditions, where aiming points for heliostats will not be limited to pre-set locations. This will be the viewpoint (and

\footnotetext{
* Corresponding author at: Instituto de Matemáticas de la Universidad de Sevilla (IMUS), Edificio Celestino Mutis - 1 Planta, Avda. Reina Mercedes, s/n, 41012 Sevilla, Spain.

E-mail addresses: tashley@us.es (T. Ashley), ecarrizosa@us.es (E. Carrizosa), cara@us.es (E. Fernández-Cara).
} 


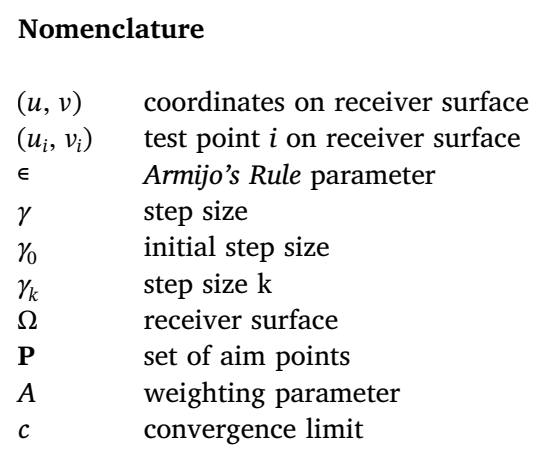

$\begin{array}{ll}E^{t a r} & \text { target distribution } \\ E_{u, v}^{t a r} & \text { target distribution at point }(u, v) \\ f(h, p) & \text { total radiation on receiver for heliostat } h \text { aiming at } p \\ F_{u, v}(h, p) & \text { Gaussian distribution of heliostat } h \text { aiming at } p \\ g & \text { objective function } \\ H & \text { total number of heliostats in field } \\ h & \text { heliostat } h \\ I & \text { number of test points } \\ i & \text { receiver test point } \\ p & \text { aiming point } p \\ p_{h} & \text { aiming point for heliostat } h\end{array}$

$E^{\text {tar }}$

$E_{u, v}^{l a r}$

$F_{u, v}(h, p)$

$g$

H

$h$

$p$

$p_{h}$ target distribution

target distribution at point $(u, v)$

Gaussian distribution of heliostat $h$ aiming at $p$

total number of heliostats in field

heliostat $h$

receiver test point

aiming point for heliostat $h$ the main contribution) in this paper.

Whilst the first objective is the maximisation of total radiation reaching the receiver surface, operational limitations of the SPT plant must be taken into consideration. As previously noted, these include inhomogeneous heating of the receiver surface, where large thermal fluxes can cause non-optimal energy generation, or even permanent damage to the receiver components. Hence, the objective in this work is to maximise the radiation captured by the SPT plant receiver, whilst taking into account the deviance from a desired radiation distribution across the receiver using continuous optimisation models. The two objectives will be combined into one single non-convex, non-linear criterion via additive weighting. By varying the weights, an approximation to the corresponding Pareto Front will be obtained. Some related heuristic methods can be found in Wagner and Wendelin (2016).

The structure of this paper is as follows: Section 2 details the problem formulation; Section 3 describes how to numerically formulate the problem; Section 4 provides an illustrative example of the construction of the Pareto frontier of the bi-objective problem, and Section 5 presents concluding remarks.

\section{Model}

The first component of the criterion in our aiming strategy optimisation model is the radiation generated by the heliostats on the receiver surface.

As in Ashley et al. (2017), the radiation passing through the system is modelled using a Gaussian distribution on the receiver, a non-empty bounded open convex set $\Omega \subset \mathbb{R}^{2}$. We will denote by $|\Omega|$ (resp. $\bar{\Omega}$ ) the measure (resp. the closure) of $\Omega$. In this paper, any heliostat $h \in H$ will be required to aim at point $p_{h} \in \bar{\Omega}$. The value of this distribution evaluated at a cartesian point $(u, v) \in \Omega$ is denoted by $F_{u, v}(h, p)$, where the total radiation at any point $(u, v)$ is the sum of contributions from all heliostats.

In this work, the receiver is assumed to be a circular flat plate. This simplification is utilised for convenience in the calculations, however it should be noted that the optimisation model and numerical methods developed in this paper are directly extendible to general realistic receiver designs.

The total radiation captured over the whole receiver surface associated to heliostat $h$ aiming at point $p_{h} \in \bar{\Omega}$ can be written as $f\left(h, p_{h}\right)=\int F_{u, v}\left(h, p_{h}\right) d \Omega$. Therefore the total radiation corresponding to all heliostats in $H$ can be expressed in the form:

$\sum_{h \in H} f\left(h, p_{h}\right)$

The second criterion in this problem considers the difference between the radiation reaching the receiver and a desired target distribution $E_{u, v}^{t a r}$. This distribution will in practise be decided by the SPT plant operators, depending on weather conditions and the thermal status of the receiver. This second objective can be expressed as the integral of the square of the calculated radiation $F_{u, v}\left(h, p_{h}\right)$ minus the target distribution $E_{u, v}^{\text {tar }}$ :

$\int\left[\sum_{h \in H} F_{u, v}\left(h, p_{h}\right)-E_{u, v}^{t a r}\right]^{2} d \Omega$.

Other criteria, such as the overall radiation excess with respect to a target distribution, may be considered instead. The reason to choose (2) in this paper is that, as seen below, it leads to a continuously differentiable objective function, which allows us to consider gradient based algorithms for the problem resolution.

Combining (1) and (2), we arrive at a suitable objective function, where we consider a parameter $A \in(0,1)$ that controls the relative importance between maximising energy and minimising deviation from the target distribution:

$A \sum_{h \in H} f\left(h, p_{h}\right)-(1-A) \int\left[\sum_{h \in H} F_{u, v}\left(h, p_{h}\right)-E_{u, v}^{t a r}\right]^{2} d \Omega$.

which is to be maximised.

Optimising the objective function in (3) over a range of values of $A$ produces an approximation to the Pareto front of the bi-objective problem of simultaneous optimisation of energy generated and deviation with respect to the target distribution.

The next section of this work devises a numerical method for the solution of the continuous optimisation model proposed in this Section.

\section{Numerical methods}

The continuous optimisation model proposed in Section 2 involves calculating integrals over the receiver surface $\Omega$. For numerical purposes, these integrals must be replaced by summations over a finite set of equally spaced test points $\left(u_{i}, v_{i}\right) \in \Omega$ with $i=1, \ldots, I$. The size of $I$ directly affects the precision to which the problem in Eq. (3) is approximated and, also, affects the numerical complexity of the problem solution. The deviation from the desired flux distribution $E_{u, v}^{t a r}$ is then calculated and furnished only for the test points $\left(u_{i}, v_{i}\right)$. It is important to note that we 'discretise' the receiver $\Omega$ only at the numerical integration level (remember that $p_{h}$ can take any value in $\Omega$ ).

The resulting objective function is the following:

$g(\mathbf{P}):=A \sum_{h \in H} f_{s p}\left(h, p_{h}\right)-(1-A) \frac{|\Omega|}{I} \sum_{i=1}^{I}\left[\sum_{h \in H} F_{u_{i}, v_{i}}\left(h, p_{h}\right)-E_{u_{i}, v_{i}}^{t a r}\right]^{2}$,

where $\mathbf{P}=\left(p_{h}\right) h \in H,|\Omega|$ denotes the measure of $\Omega$ and $|I|$ is the cardinality of $I$.

The coefficient $\frac{|\Omega|}{I}$ stems from the numerical approximation of the integral in (3).

Thus, we want to solve a non-linear non-convex optimisation problem with continuous variables of large dimension (twice the number of heliostats in the field), subject to the convex constraints $p_{h} \in \bar{\Omega}$.

Function $g$ in Eq. (4) can then be maximised using a gradient ascent 
algorithm with projection, see Le Floch et al. (2015, 2011). We start with an initial solution $\mathbf{P}_{0}$ and then, at each iteration of the algorithm, update the components of $\mathbf{P}$ in the direction of steepest ascent of the objective function $g$ by step size $\gamma$. The final step in each iteration of the algorithm utilises a projection method to correct any values of $\mathbf{P}$ to ensure heliostats aim at $\Omega$.

The selection of the stepsize $\gamma$ taken at each update to the gradient ascent algorithm is an important factor in the convergence and much research has focused on this choice, for example Liu and Liu (2018). If the stepsize is too large, the algorithm may diverge, and if it is too small, it will take too long to converge. A method to find the optimal stepsize at each iteration can be found using Armijo's Rule (McCormick, 1977), where a constant value $\in \in(0,1)$ is used to iteratively reduce the stepsize until an improvement on the objective function is no longer given.

This is tested at the $k$-th iteration of the algorithm against the $(k-1)$-th iteration as follows:

$g\left(\mathbf{P}_{k}\right)>g\left(\mathbf{P}_{k-1}\right)$

Traditionally, the value of $\gamma$ is fixed for all elements of the system. However, in this work the algorithm is adapted to allow different $\gamma$ for each heliostat $h$ :

$\gamma_{k, h}=\gamma_{k-1, h} \cdot \epsilon$.

This permits heliostats to take the greatest stepsize independently of each other, thereby potentially increasing the speed of the algorithm. However, it is also important to note that this procedure may in fact increase running times, so careful selection of $\epsilon$ is required.

Therefore, the gradient ascent algorithm reads:

$\widetilde{\mathbf{P}}_{k+1}=\mathbf{P}_{k}+\gamma_{k, h} \nabla g\left(\mathbf{P}_{k}\right), \quad \mathbf{P}_{k+1}=P\left(\widetilde{\mathbf{P}}_{k+1}\right)$,

where for each $\mathbf{P}, P(\mathbf{P})$ denotes the component-wise projection of $\mathbf{P}$ onto $\Omega$.

The considered objective function and its gradient are complex. It is therefore important to customise related effective numerical techniques leading to reasonable computational effort.

The function being modelled in this paper is highly multi-modal and, consequently, convergence to the global optimum is significantly dependent on the starting $\mathbf{P}_{0}$ given to the algorithm. We therefore apply a multistart procedure where the algorithm is run multiple times, utilising a different random allocation of starting heliostat aiming points. Applying this method for each value of $A$, it is possible to approximate the Pareto front of the model.

It will be accepted that the aforementioned algorithm converges to a local optimum when the Euclidean norm of the gradient of the energy function is below a selected precision value $c$. The selection of this value determines how close to the local optimum the algorithm must finish, whilst also heavily influencing the computation time required.

Summarising, the convergence test used in the algorithm can be written in the form

$\|\nabla g(\mathbf{P})\| \leqslant c$,

where

$\nabla g(\mathbf{P})=A \sum_{h \in H} \nabla f^{s p}\left(h, p_{h}\right)$

$-\frac{2(1-A)|\Omega|}{I} \sum_{i=1}^{I}\left[\left(\sum_{h \in H} F_{u_{i}, v_{i}}\left(h, p_{h}\right)-E_{u_{i}, v_{i}}^{t a r}\right) \sum_{h \in H} \nabla F^{u_{i}, v_{i}}\left(h, p_{h}\right)\right]$.

Note that this approach can serve to consider many prescribed flux distributions $F_{u, v}$ (not necessarily Gaussian).

In the following section we apply the numerical discretisation of the continuous optimisation model developed in this paper to a real SPT plant and we present an illustrative example.

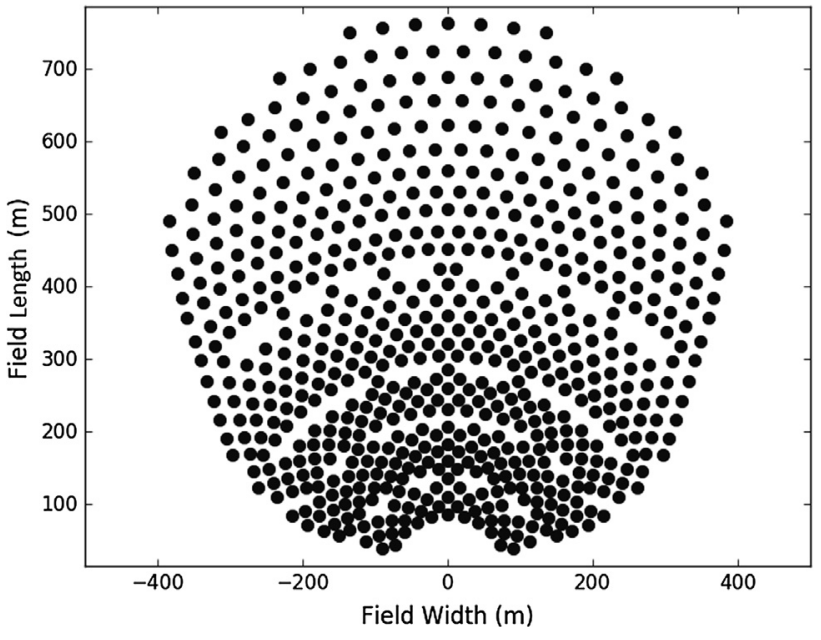

Fig. 1. Heliostat locations.

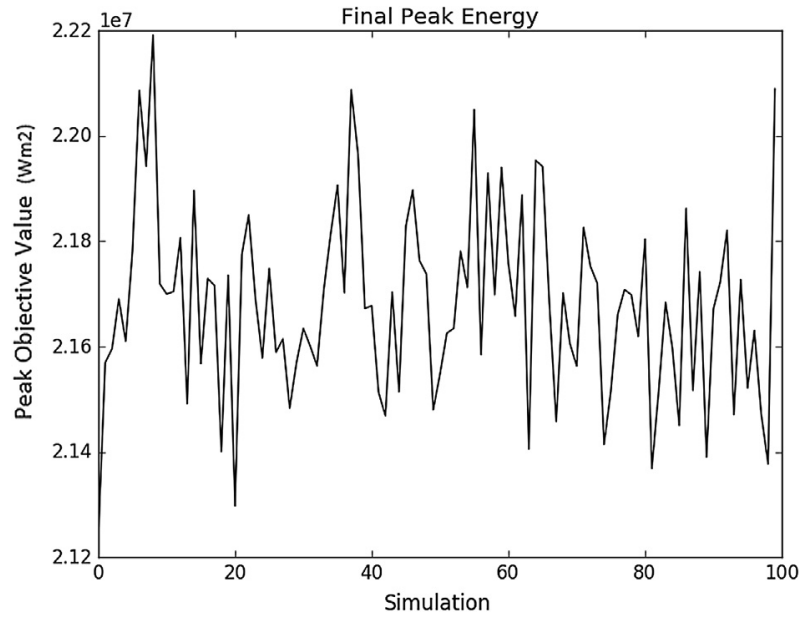

Fig. 2. Multistart analysis.

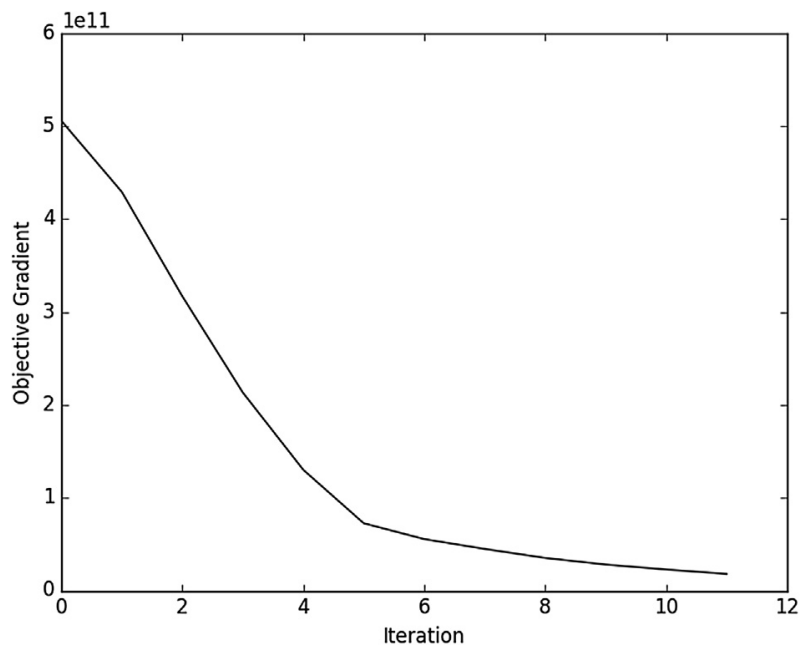

Fig. 3. Gradient convergence.

\section{Illustrative example}

The developed optimisation procedure is illustrated using the PS10 SPT plant in Sanlúcar la Mayor, Seville (Abengoa PS10 SPT Plant). This SPT plant has a field of 624 heliostats in a South facing field, arranged as shown in Fig. 1. 


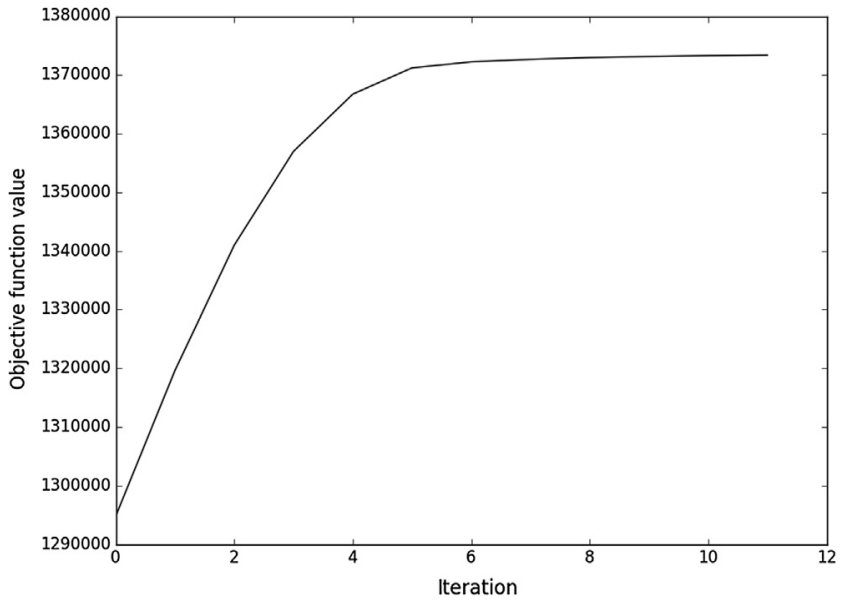

Fig. 4. Objective function convergence.

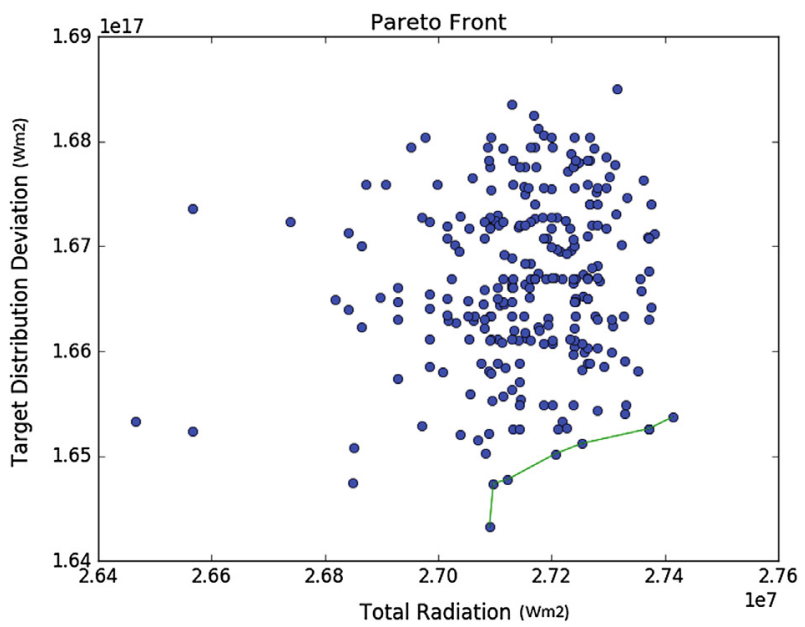

Fig. 5. Objective values with Pareto Front.

The receiver $\Omega$ is modelled as a disk and the grid points $\left(u_{i}, v_{i}\right)$ have been fixed equally spaced and of equal number in both dimensions. However, the following arguments and techniques are equally valid for any other 2D domain. Essentially, only the integrals in (2) and (3) will be affected by the geometrical properties of $\Omega$.

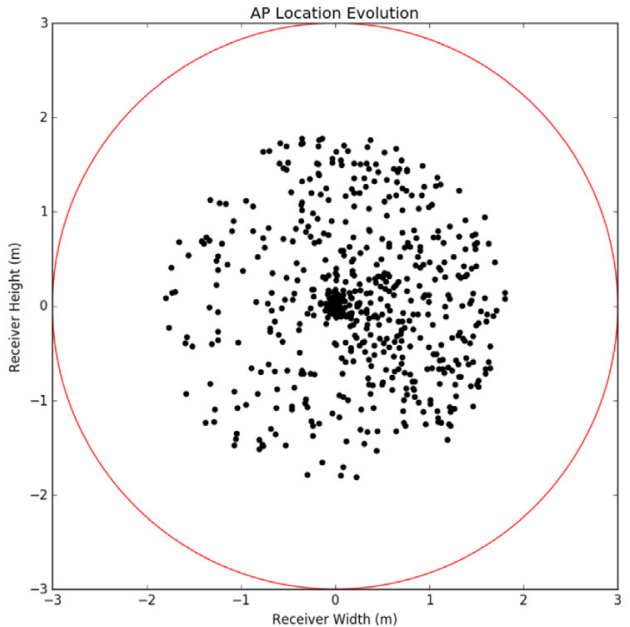

(a) Optimised aiming strategy
The algorithm developed in Section 3 is implemented in Python on a standard specification desktop computer, for a chosen time point of midday.

The Armijo's Rule parameter $\epsilon$ is set to a value of 0.8 , as the suggested value in McCormick (1977), and the initial value for $\gamma$ is set to 0.01 . A lower limit of $\gamma$ equal to $10^{-8}$ is set, in order to prevent unnecessary computations occurring in the algorithm.

As mentioned above, since the problem is highly multi-modal, a multistart procedure has been implemented, where the best solution across 30 runs is selected for each variation of the parameter $A$.

Fig. 2 shows the peak of the objective function for one value of $A$ across 100 multistart runs, where the multi-modality of the problem can be clearly seen. The variance in solutions found over the 100 multistart runs is also affected by the stop criterion and step size used in each application of the algorithm, meaning that whilst only a few solutions may be found, the level of convergence may not be the same. This can be seen in Fig. 2 by the number of peaks and troughs at similar, but slightly different, values. These possibly represent the same solution, reached at different levels of convergence. Therefore, when utilising an adequate selection of step size and convergence test, it is found that a multistart operation with 30 runs suffices in practise to find a solution. The convergence of the objective function to the solution, and the gradient to zero, can be seen in Figs. 3 and 4 for one particular simulation.

The parameter $A$ in the objective function in Eq. (4) has been tested between 0 and 1 in steps of 0.01 . A value of 0 indicates that the absence of deviation from the target distribution objective is the most important contribution. Contrarily, a value of 1 indicates that the total radiation objective is the most relevant. The target distribution has been assumed constant across the receiver surface. It is important to note that the chosen target distribution must be tailored to the SPT plants current conditions, and a constant distribution has been implemented here as an example.

The values of both objectives for each value of $A$ are shown in Fig. 5, where the Pareto Front has also been marked. The set of Pareto equilibria identifies those solutions which cannot be improved in terms of both objectives, and therefore give the best optima in terms of minimising target distribution deviation and maximising total radiation capture.

The results shown in Fig. 5 indicate that the choice of $A$ can produce highly differing results in the objective function, and this can generate quite different aiming strategies trading off energy maximisation and minimisation of deviation from the target radiation distribution. This is

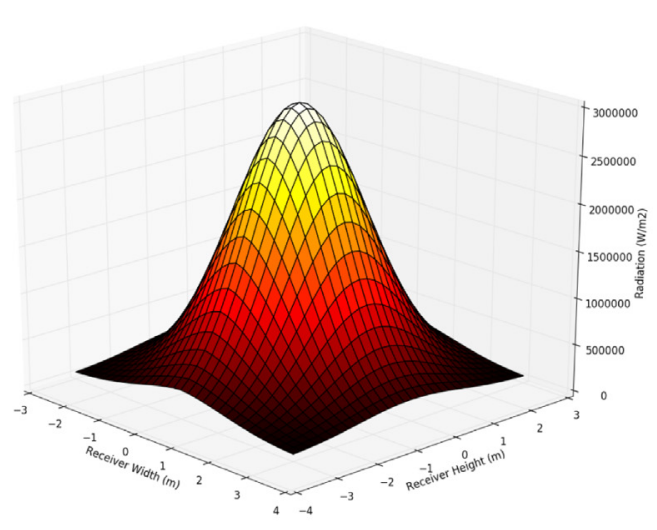

(b) Optimised flux distribution with maximum flux $2.8 \mathrm{e} 6$

Fig. 6. Optimised result with $A=0.9$. 


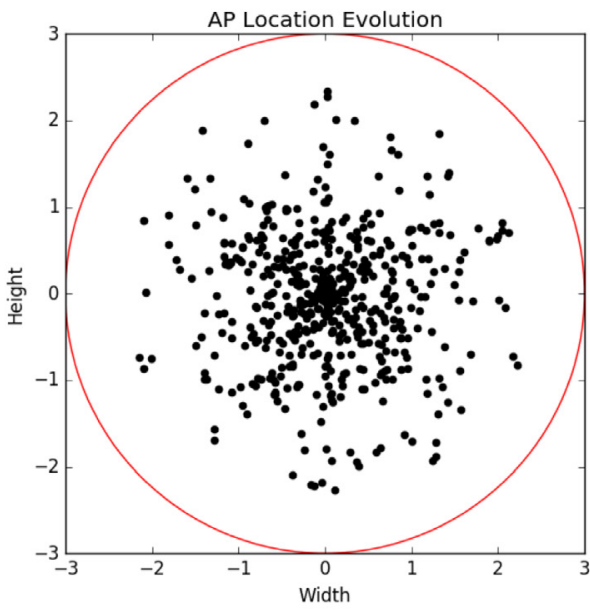

(a) Optimised aiming strategy

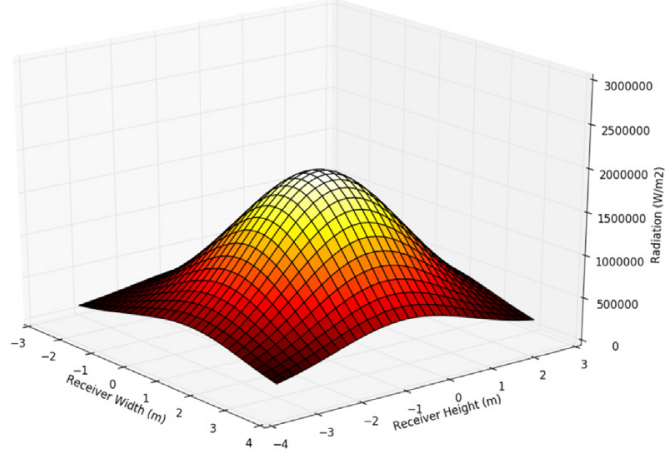

(b) Optimised flux distribution with maximum flux $2 \mathrm{e} 6$

Fig. 7. Optimised result with $A=0.3$.

illustrated in Figs. $6 \mathrm{a}$ and $7 \mathrm{~b}$.

Fig. 6a shows the resultant aiming strategy when the value of $A$ is set to 0.9 , causing the captured radiation to be significantly more important than adhering to the target distribution. The aiming strategy will capture more radiation, as shown in Fig. 6b, but will fail to yield a homogeneous flux distribution, due to the centrally focused heliostats.

Fig. 7a shows the resultant aiming strategy when $A$ is set to 0.3 , which creates a much more homogeneous flux distribution, as shown in Fig. 7b. These Figures demonstrate the importance of the value of making a judicious choice of $A$, since improvement in both objectives is highly dependent on its choice.

The results illustrated in this section are for one particular time point, and two examples of the weighting variable $A$. Each run of this simulation takes less than $10 \mathrm{~s}$, which is then multiplied by the number of multistarts performed. With advance knowledge of local weather conditions and predictable solar radiation input, the optimal aiming strategy can be calculated across a day. However, to account for rapid changes in weather, it may be useful to re-calculate the optimal aiming strategy in short time scales. Therefore, the rapid computation of this algorithm is advantageous, and also lends itself to applications in SPT plants with larger heliostat field sizes.

The next section draws conclusions from the method and numerical illustration developed in this paper, and also discusses possible extensions and current research.

\section{Further comments, extensions and conclusions}

In this work, a bi-objective optimisation model has been implemented to find the optimal aiming strategy for a SPT plant of any size or shape, and a numerical illustration for a real SPT plant is presented.

For the PS10 SPT plant with a field of 624 heliostats, the optimal aiming strategy has been found using the objective function given in (4), that must be viewed as a numerical approximation to (3). In this illustrative example, we have solved the optimisation problem with a multistart procedure for multiple values of the parameter $A$ in $(0,1)$.

Utilising the optimisation model and numerical method developed in this work, it is possible for the operators of a SPT plant to identify the optimal aiming strategy, considering current weather and plant requirements. Depending on the choice of the weighting parameter $A$ in the objective function, it is possible to seek a desired balance between maximising overall radiation captured and minimising deviation from a desired distribution across the receiver.

The method developed in this work significantly improves upon the method presented by the authors in Ashley et al. (2017), as here the problem space is not constrained by pre-set aiming points, and allows for realistic representation of SPT plant operations, where it is possible to monitor relative performance of each objective.

The numerical method developed for this work made use of Armijo's rule to iterate the step-size in the algorithm, with independent stepsizes for all components in the set space. This modification can cause longer run times for the simulation. However, a careful application of the algorithm in this case allows it to improve performance and converge to the optimal solution. This performance increase over other methods is of critical importance when simulations in a SPT plant will be re-run during the course of a day for changing local weather conditions and solar input.

For the PS10 SPT plant, the optimal aiming strategy can be easily found using the method outlined in this work. For larger SPT plants with more heliostats in the field, the same arguments and techniques can be applied. However, if the number of heliostats is large, and the associated computational cost becomes untenable, an amendment can be made to reduce the problem dimensionality. A possible modification could be the use of a clustering algorithm, such as in Ashley et al. (2017) and Carrizosa et al. (2013). In that research, the heliostats in the field are clustered using an optimisation procedure which takes into account potential radiation generation as well as physical location and, then, the same aiming strategy is chosen for all heliostats within the same cluster.

The performance of aiming strategies found utilising the method presented in this article should be compared against similar methods by other authors, for instance Collado and Guallar (2019). There, the authors consider a two parameter method for reduction of peak flux levels on the receiver, and compare against the method given in Vant-Hull (2002), which utilised a single parameter method to divide flux peaks into two and reduced maximum load.

Another extension to this work that could assist in reducing computational cost is the application of stochastic techniques Fonseca et al. (2017), Schmidt et al. (2013), and Wang (2017) where, at each step, the gradient is calculated for just a random sample of heliostats and then used to update the general population. Such methods reduce the calculation time required for each iteration of the algorithm at the cost of utilising several (or many) potentially erroneous components of the gradients.

The method presented in this paper is static in time, and therefore does not consider how the optimal aiming strategy would change as the incident radiation on the heliostat field varies across a day. The authors are developing an optimisation model for the dynamic case of this 
problem, where the bi-objective function is adapted to account for additional time evolution constraints.

\section{Acknowledgements}

This research is being supported by the Spanish Government through the research project PCIN-2015-108 and is being conducted within the group MTM2015-65915-R at the University of Seville.

\section{References}

Abengoa PS10 SPT Plant. Abengoa PS10 SPT Plant < http://www.abengoasolar.com/ web/en/plantas_solares/plantas_para_terceros/espana/index.htm > (accessed: 22/ 02/2017).

Abu-hamdeh, Nidal H., Alnefaie, Khaled A., 2016. Design considerations and construction of an experimental prototype of concentrating solar power tower system in Saudi Arabia. 117, 63-65.

Ashley, Thomas, Carrizosa, Emilio, Fernández-Cara, Enrique, 2017. Optimisation of aiming strategies in Solar Power Tower plants. Energy. https://doi.org/10.1016/j. energy.2017.06.163. ISSN 03605442.

Astolfi, Marco, Binotti, Marco, Mazzola, Simone, Zanellato, Luca, Manzolini, Giampaolo, 2016. Heliostat aiming point optimization for external tower receiver. Solar Energy 1-16. https://doi.org/10.1016/j.solener.2016.03.042. ISSN 0038-092X.

Baños, R., Manzano-Agugliaro, F., Montoya, F.G., Gil, C., Alcayde, A., Gómez, J., 2011. Optimization methods applied to renewable and sustainable energy: a review. Renew. Sustain. Energy Rev. 15 (4), 1753-1766. https://doi.org/10.1016/j.rser. 2010.12.008. ISSN 13640321.

Belhomme, Boris, Pitz-Paal, Robert, Schwarzbözl, Peter, 2013. Optimization of heliostat aim point selection for central receiver systems based on the ant colony optimization metaheuristic. J. Solar Energy Eng. 136 (February 2014), 011005. https://doi.org/ 10.1115/1.4024738. ISSN 0199-6231. < http://solarenergyengineering. asmedigitalcollection. asme.org/article.aspx?doi $=10.1115 / 1.4024738>$.

Carrizosa, E., Domínguez-Bravo, C., Fernández-Cara, E., Quero, M., 2015a. A heuristic method for simultaneous tower and pattern-free field optimization on solar power systems. Comp. Operat. Res. 57, 109-122. https://doi.org/10.1016/j.cor.2014.11. 017. ISSN 03050548. < http://www.sciencedirect.com/science/article/pii/ S0305054814003219>.

Carrizosa, E., Domínguez-Bravo, C., Fernández-Cara, E., Quero, M., 2015b. Optimization of multiple receivers solar power tower systems. Energy 90, 2085-2093. https://doi. org/10.1016/j.energy.2015.08.005. ISSN 03605442.

Carrizosa, E., Domínguez-Bravo, Carmen-Ana, Fernández-Cara, E., Quero, M., 2017. An optimization tool to design the field of a solar power tower plant allowing heliostats of different sizes. Int. J. Energy Res. https://doi.org/10.1002/er. ISSN 12310956.

Carrizosa, Emilio, Mladenović, Nenad, Todosijević, Raca, 2013. Variable neighborhood search for minimum sum-of-squares clustering on networks. Euro. J. Operat. Res. 230 (2), 356-363. https://doi.org/10.1016/j.ejor.2013.04.027. ISSN 03772217.

Collado, Francisco J., Guallar, Jesus, 2019. A two-parameter aiming strategy to reduce and flatten the flux map in solar power tower plants. Solar Energy 188 (January),
185-189. https://doi.org/10.1016/j.solener.2019.06.001. ISSN 0038092X.

Cruz, N.C., Redondo, J.L., Álvarez, J.D., Berenguel, M., Ortigosa, P.M., 2016. A parallel Teaching-Learning-Based Optimization procedure for automatic heliostat aiming. J. Supercomput. https://doi.org/10.1007/s11227-016-1914-5. ISSN 0920-8542. http://link.springer.com/10.1007/s11227-016-1914-5.

Fonseca, Rahul Rahul Mark, Chen, Bailian, Jansen, Jan Dirk, Reynolds, Albert, 2017. A Stochastic Simplex Approximate Gradient (StoSAG) for optimization under uncertainty. Int. J. Numer. Meth. Eng. 109 (13), 1756-1776. https://doi.org/10.1002/ nme.5342. ISSN 10970207.

Le Floch, Caroline, Belletti, Francois, Saxena, Samveg, Bayen, Alexandre M., Moura, Scott, 2015. Distributed optimal charging of electric vehicles for demand response and load shaping. In: 54rd IEEE Proceedings of the IEEE Conference on Decision and Control (CDC), pp. 6570-6576. https://doi.org/10.1109/CDC.2015.7403254. ISSN 07431546.

Liu, Zexian, Liu, Hongwei, 2018. An efficient gradient method with approximate optimal stepsize for large-scale unconstrained optimization. Numer. Algor. 78 (1), 21-39. https://doi.org/10.1007/s11075-017-0365-2. ISSN 15729265

McCormick, Garth P., 1977. A modification of Armijo's step-size rule for negative curvature. Math. Program. 13, 111-115.

Morais, Hugo, Kádár, Péter, Faria, Pedro, Vale, Zita A., Khodr, H.M., 2010. Optimal scheduling of a renewable micro-grid in an isolated load area using mixed-integer linear programming. Renew. Energy 35 (1), 151-156. https://doi.org/10.1016/j. renene.2009.02.031. ISSN 09601481.

Przybylski, Anthony, Gandibleux, Xavier, 2017. Multi-objective branch and bound. Euro. J. Operat. Res. 260 (3), 856-872. https://doi.org/10.1016/J.EJOR.2017.01.032. ISSN 0377-2217. < https://www.sciencedirect.com/science/article/pii/ S037722171730067X > .

Ranganathan, Raghuram, Mikhael, Wasfy, Kutkut, Nasser, Batarseh, Issa, 2011. Adaptive sun tracking algorithm for incident energy maximization and efficiency improvement of PV panels. Renew. Energy 36 (10), 2623-2626. https://doi.org/10.1016/j.renene. 2010.06.011. ISSN 09601481.

Relloso, S., García, E., 2015. Tower technology cost reduction approach after gemasolar experience. Energy Proc. 69, 1660-1666. https://doi.org/10.1016/j.egypro.2015.03. 125. ISSN 18766102.

Schmidt Mark, Le Roux Nicolas, Bach Francis, 2013. Minimizing finite sums with the stochastic average gradient, pp. 83-112. ISSN 14364646, doi:https://doi.org/10. 1007/s10107-016-1030-6 < http://arxiv.org/abs/1309.2388 >

Tawhid, Mohamed A, Savsani, Vimal, 2018. A novel multi-objective optimization algorithm based on artificial algae for multi-objective engineering design problems. Appl. Intell. https://doi.org/10.1007/s10489-018-1170-x.

Vant-Hull, 2002. J. Solar Energy Eng. 2, 124. https://doi.org/10.1115/1.1464124.

Wagner, Michael J., Wendelin, Tim, 2018. SolarPILOT: a power tower solar field layout and characterization tool. Solar Energy 171, 185-196. https://doi.org/10.1016/j. solener.2018.06.063. ISSN 0038092X.

Wang, Honggang, 2017. Multi-objective retrospective optimization using stochastic zigzag search. Euro. J. Operat. Res. 263 (3), 946-960. https://doi.org/10.1016/j. ejor.2017.06.039. ISSN 03772217.

Qiang, Yu., Wang, Zhifeng, Ershu, Xu., 2014. Analysis and improvement of solar flux distribution inside a cavity receiver based on multi-focal points of heliostat field. Appl. Energy 136, 417-430. https://doi.org/10.1016/j.apenergy.2014.09.008. ISSN 03062619. 